\title{
Le développement des biocarburants en France : une analyse de la compétition entre colza ester et colza alimentaire à I'horizon 2010
}

Jean-Claude SOURIE

David TREGUER

INRA - Économie publique, BP 0178850 Thiverval Grignon

Tél. : 01308153 61; fax : 0130815368

<sourie@grignon.inra.fr>

Initialement développés pour compenser les revenus des agriculteurs à la suite de la mise en place de la réforme de la Politique agricole commune (PAC) de 1993 (et l'obligation d'une mise en jachère des terres pour réguler les excédents alimentaires), les biocarburants sont appelés à connaitre une deuxième phase de développement, placée sous le signe des engagements européens dans le cadre du Protocole de Kyoto.

La France s'est engagée sur le chemin d'une évolution des quantités de biocarburants tracée par la Commission européenne. Ainsi le gouvernement a annoncé au début du mois de février les nouveaux agréments de biocarburants pour 2007.

Ces agréments ont renforcé la domination de la filière ester dans le paysage des biocarburants français, qui devrait bénéficier en 2007 d'un peu moins d'un million de tonnes d'agréments, contre 420000 pour la filière éthanol. En aval de la filière (i.e. au niveau des motorisations), cet avantage ne souffre aucune contestation, tant la prépondérance des moteurs diesel est évidente.

Le développement important de la production d'ester fondé sur une culture dont la productivité par hectare est plutôt faible - 1300 litres d'ester en moyenne par hectare - va demander des surfaces de terres significatives. Actuellement, les surfaces consacrées à l'ester de colza sont de l'ordre de 300000 hectares; elles pourraient tripler dans un premier temps en 2007, puis être multipliées par 2 pour atteindre I'objectif de la Commission en 2010, à savoir $5,75 \%$ de biocarburants dans les carburants terrestres. Les surfaces nécessaires pour satisfaire les besoins totaux en biocarburants pourraient alors dépasser 1400000 hectares en

\begin{abstract}
Following the EU directives which aim at developing biofuels up to $5.75 \%$ of the total fuels used for terrestrial transportation purposes, the French government has announced that a supplementary 800000 tons' agreement of biofuels will be produced by 2007. Rapeseed Methyl Ester (RME, mixed with diesel oil) will then be produced up to nearly 1 Million tons. Particular emphasis is dedicated in this article to the competition between food and energy rapeseed. Thanks to a linear programming model, insights can be given about the evolutions between the different rapeseed areas. Four different scenarios are developed in order to enlighten some aspects of this competition.
\end{abstract}

Key words: biofuels, set-aside, rapeseed, food, energy, linear programming
2010, dont 1100000 hectares d'oléagineux (tableau 1).

La France dispose de 1,5 millions d'hectares de jachère dont seulement $70 \%$ environ sont exploitables dans de bonnes conditions. Tout le gel de terre ne pourra pas accueillir ces surfaces.

Pour étudier l'économie des biocarburants, I'INRA dispose d'un modèle d'offre agricole séquentiel OSCAR ${ }^{1}$ tenant compte de la diversité des exploitations et de leurs contraintes agronomiques, du progrès agronomique et des évolutions des politiques agricole et sucrière (découplage, évolution du régime des quotas). Ce modèle maximise les revenus agricoles des producteurs sous contraintes, sur une période allant de 2003 à 2010. En interrogeant ce modèle pour un cadre d'hypothèses économique et politique, on peut anticiper les effets de la production d'ester sur l'offre de colza alimentaire, non alimentaire et sur les coûts du colza ester. Les mécanismes microéconomiques du modèle qui concernent plus précisé-

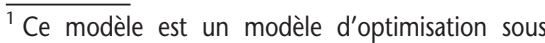
contraintes, formé de 1300 modèles élémentaires d'exploitations extraites du RICA.
}

ment le colza sont décrits dans le paragraphe suivant.

\section{Comportement économique de producteurs agricoles et offre de colza énergétique}

Le colza énergétique est une opportunité parmi d'autres pour le producteur agricole. Pour que cette culture soit introduite dans un assolement ${ }^{2}$, elle doit apporter un revenu agricole additionnel. Cette production permettra de valoriser des équipements présents sur l'exploitation ainsi que le travail disponible, familial ou salarié. La surface des colzas alimentaires et non alimentaires ne dépasse pas généralement $30 \%$ de la SAU pour des raisons agronomiques. Cette contrainte peut d'ailleurs amener assez rapidement une compétition entre I'alimentaire et le non alimentaire dans

\footnotetext{
${ }^{2}$ Le revenu agricole est la différence entre les recettes augmentées des primes PAC et les charges, variables et fixes. À partir de ce revenu agricole, l'exploitant obtient son salaire et les moyens pour autofinancer la croissance de son exploitation, d'où l'importance du revenu en tant que critère de gestion.
}

Tableau 1. Estimations des évolutions de surfaces en cultures énergétiques.

\begin{tabular}{|c|c|c|c|c|c|}
\hline Années & Colza & Betterave & Blé & Total & $\begin{array}{c}\text { Gel PAC } \\
\text { disponible }\end{array}$ \\
\hline & 324 & 10 & 20 & 350 & 1050 \\
\hline 2005 & 426 & 20 & 40 & 480 & 1050 \\
\hline 2007 & 769 & 42 & 84 & 890 & 1050 \\
\hline 2010 & 1100 * & \multicolumn{2}{|c|}{300} & 1400 & 1050 \\
\hline
\end{tabular}

* rendement moyen en colza $37 \mathrm{q}$. 
les régions (périphérie Est du Bassin parisien par exemple) où les assolements sont fortement chargés en colza alimentaire, par manque de têtes d'assolement.

Soucieux d'optimiser son revenu, le producteur commencera à produire du colza à la place de la jachère PAC, instaurée en 1993 pour réguler l'offre agricole et réduire les soutiens à I'agriculture. Ce faisant, l'agriculteur continuera à bénéficier de l'aide directe à la jachère, au moins jusqu'en 2006 avant le découplage. Ce choix est en général préférable à celui qui consiste à réduire d'emblée la surface en colza alimentaire pour bénéficier de l'avantage de 45 euros par hectare et à maintenir inchangée la surface en jachère. II suffit en effet que la recette apportée par le colza soit supérieure aux charges variables (semences, engrais, traitements, carburants et entretien des matériels) pour que cette production génère un revenu agricole additionnel. Cette relation peut être satisfaite pour un prix seuil du colza très inférieur au prix du colza alimentaire ; c'est pourquoi durant les premières années de production de colza énergétique, le prix du colza énergétique était très inférieur au prix du colza alimentaire.

La présence de jachère crée donc un contexte favorable à la production de colza et plus généralement à la production de toute culture énergétique : blé et betterave notamment. II faut toutefois que les terres en jachère soient d'une fertilité proche de celles en culture ; 70 à $75 \%$ des terres en jachère seraient dans ce cas. La présence de cette jachère va bien entendu freiner dans un premier temps la compétition directe avec le colza alimentaire mais on verra que cette compétition va se manifester nettement avant d'atteindre le seuil de 1 million $d^{\prime}$ hectares de jachères cultivables.

Ensuite, le producteur aura intérêt à réduire sa production de colza alimentaire pour satisfaire son contrat de production si le prix du colza énergétique est au moins égal au prix du colza alimentaire ; le producteur va alors empocher la prime de 45 euros par hectare qui constituera son revenu additionnel; ce revenu est nettement inférieur en moyenne à celui obtenu en produisant du colza sur jachère, soit 190 euros en moyenne pour un prix du colza producteur égal à 19 euros par quintal. Logiquement, on devrait assister alors à une réduction des exportations de graines de colza puisque la France est exportatrice nette. Certains pensent que l'accroissement de la production de colza pourrait réduire la production de céréales et donc les exportations. Ce mécanisme n'est pas compatible avec les règles d'une gestion économique rationnelle de l'assolement basées sur l'optimisation du revenu agricole.

\section{Compétition entre colza alimentaire et colza non alimentaire}

\section{Définitions des scénarios et hypothèses}

Les conséquences de la politique de développement des biocarburants sur l'offre de colza alimentaire et sur les coûts de l'ester vont être étudiées sur la période 2004-2010. Les offres de colza, de blé et de betterave énergétiques augmentent parallèlement. Ces cultures sont toutes en compétition pour l'usage des sols en jachère et cultivés. C'est le modèle utilisé qui, en minimisant les coûts, répartit les productions énergétiques de manière optimale entre les exploitations et entre la partie cultivée et la partie en jachère de chaque exploitation.

Les colzas sont techniquement identiques: même rendement, même itinéraire technique et mêmes charges variables. En plus des charges variables classiques, l'entretien des matériels de culture ainsi que les carburants sont comptabilisés dans ces charges, ce qui défavorise relativement le colza ester sur jachère par rapport à la jachère. Au fil des années, le rendement des colzas va s'accroître de 0,3 quintal par hectare et par an, celui-ci passant en moyenne de 35,5 à 37,2 quintaux par hectare. Une partie de ce progrès (20\%) est consommée par un accroissement de charges variables. La jachère et le colza sont des têtes d'assolement ayant des effets identiques sur le blé de la rotation.

En 2006 va s'appliquer le découplage partiel des aides; en outre, on suppose une évolution du régime sucre à la même date, provoquant une baisse des quotas (baisse de $33 \%$ du quota $A+B$ ) et des prix.

Le découplage des aides, qu'il soit partiel ou total, ne va pas modifier la compétitivité relative des colzas hors jachère et sur jachère puisque les aides PAC sont les mêmes par hectare de jachère ou par hectare de colza alimentaire. Par contre, la prime de 45 euros par hectare renforce la compétitivité du colza ester dans I'assolement alimentaire. Cet élément sera isolé en tant que paramètre des scénarios. L'évolution de l'OCM sucre va dans un premier temps diminuer les têtes d'assolement en régions betteravières et favoriser ainsi le colza ; cependant, dans un deuxième temps la sole betteravière pourrait s'accroître en raison de la compétitivité de la France au sein de l'Europe et le colza pourrait alors en pâtir. Dans les simulations effectuées, la première hypothèse sera uniquement retenue.

Quatre scénarios vont être étudiés pour tester leurs effets sur l'offre alimentaire de colza et sur le coût de l'ester :

- du scénario central A ;

- et des variantes concernant :

- la prime spécifique à la culture énergétique : scénario $A$ et $B$,

- une stabilisation des prix du colza alimentaire par rapport à une croissance de ces prix, les prix des autres cultures restant inchangés, prix des céréales notamment : scénario $A$ et $C$;

- la suppression jachère: scénarios $A$ et $D$ (tableau 2).

\section{Analyse détaillée du scénario Central A (figure 1)}

- La compétition entre alimentaire et non alimentaire se manifeste assez rapidement pour 470000 hectares de colza.

Jusqu'en 2006, il n'y a pas de compétition entre le colza non alimentaire et le colza alimentaire. Ce dernier s'accroît sous l'effet du découplage et de la réforme de l'OCM sucre et également en raison de l'évolution favorable de ses prix comparés à ceux des céréales. La compétition commence à se manifester en 2006 pour une production de 700000 tonnes d'ester, soit 470000 hectares de colza sur jachère environ. La surface en colza alimentaire commence alors à décroître régulièrement jusqu'en 2010, date à laquelle la surface devient inférieure à la surface initiale. Par rapport au sommet de 2006, 400000 hectares de colza alimentaire sont déplacés par le colza non alimentaire. Globalement, les surfaces en colza alimentaire et non alimentaire s'accroissent fortement jusqu'en 2006 puis continuent à s'accroître jusqu'en 2010 mais à un rythme plus lent. Cette compétition précoce entre colzas alimentaire et non alimentaire découle de plusieurs raisons : $25 \%$ de jachère est constituée de terres non cultivables (qualité des sols, éloignement), le blé et la betterave éthanol occupent également 280000 hectares de jachère, enfin, I'aide de 45 euros favorise un

Tableau 2. Caractéristiques des principaux scénarios simulés.

\begin{tabular}{|lcccccc|}
\hline Scénarios & $\begin{array}{c}\text { Taux de } \\
\text { jachère (\%) }\end{array}$ & $\begin{array}{c}\text { Prime colza énergétique } \\
\text { hors jachère }\end{array}$ & \multicolumn{2}{c|}{$\begin{array}{c}\text { Prix du colza } \\
€ / \mathbf{q}\end{array}$} & $\begin{array}{c}\text { Prix du blé } \\
€ / \mathbf{q}\end{array}$ \\
\hline & & & 2004 & 2010 & 2004 & 2010 \\
A & 10 & 45 & 19,2 & 19,8 & 9,2 & 8,6 \\
B & 10 & 0 & 19,2 & 19,8 & 9,2 & 8,6 \\
C & 10 & 45 & 19,2 & 18,0 & 9,2 & 8,6 \\
D & 0 & 45 & 19,2 & 19,8 & 9,2 & 8,6 \\
\hline
\end{tabular}




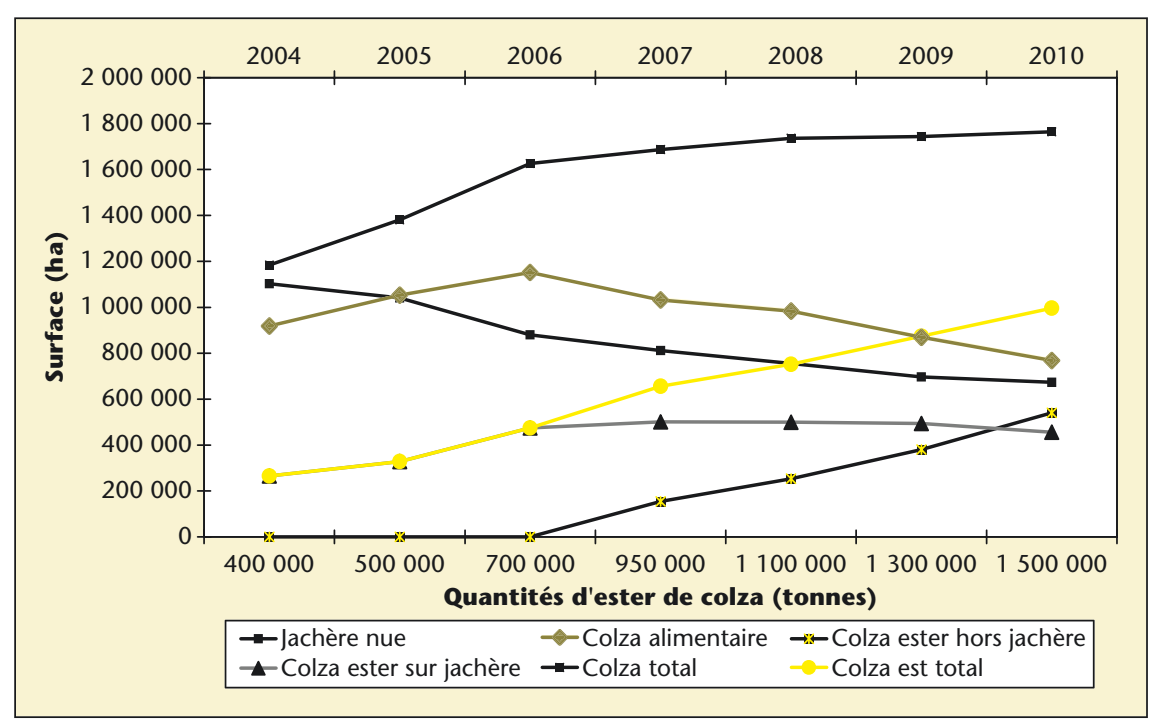

Figure 1. Evolutions des surfaces en colza alimentaire et énergétique.

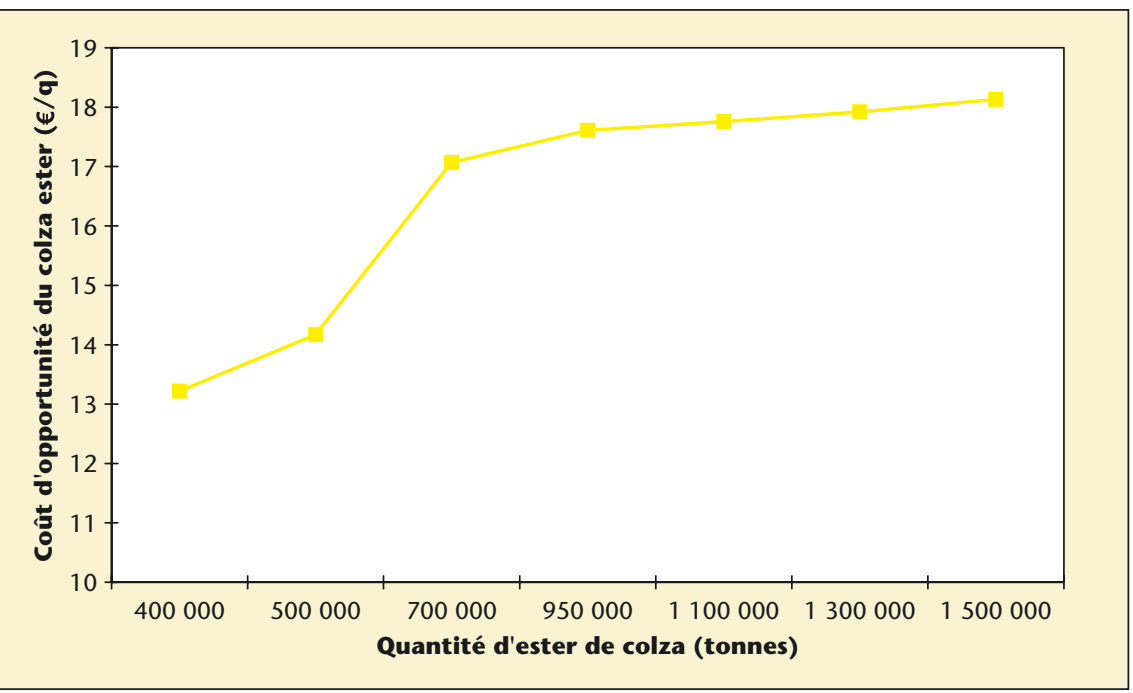

Figure 2. Coût d'opportunité du colza ester en euros par quintal.

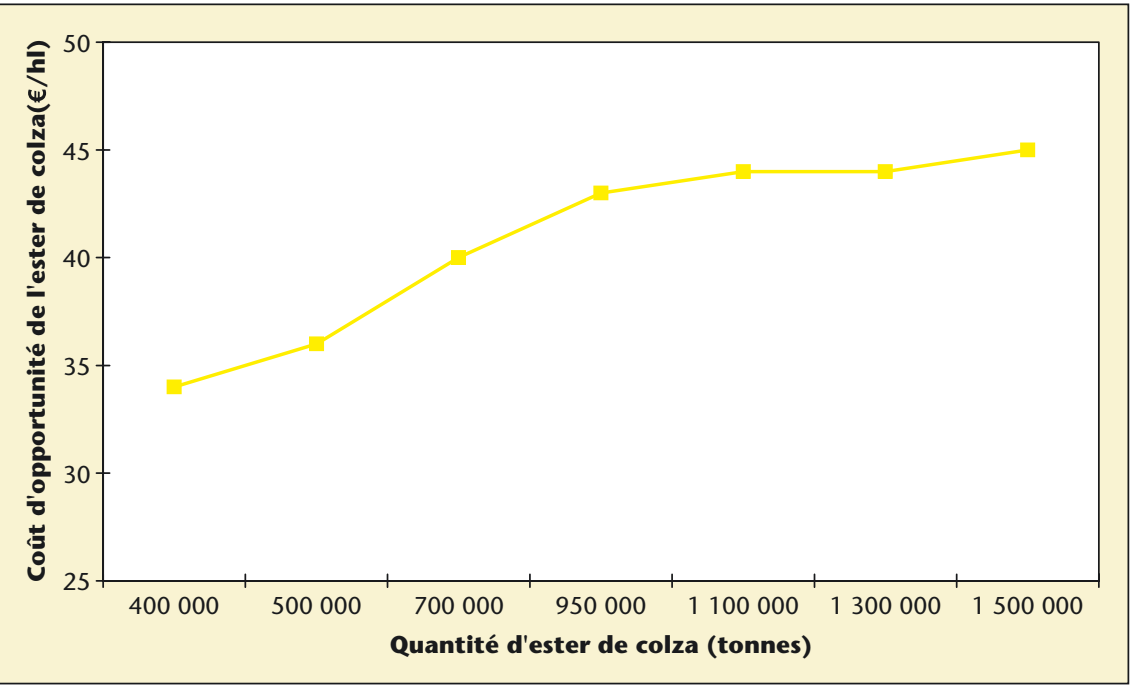

Figure 3. Coût d'opportunité de l'ester en euros par litre. glissement des cultures énergétiques de la jachère vers I'assolement alimentaire, surtout du colza plus sensible à cette aide que les deux autres cultures beaucoup plus productives par hectare.

- Compétition alimentaire et non alimentaire, coût du colza ester et de l'ester.

Cette compétition entre les colzas renchérit le colza énergétique. On observe une croissance du coût d'opportunité en fonction des hypothèses de quantités d'ester produites chaque année (figure 2).

Dans une première phase, tant que le colza se substitue à la jachère, le coût d'opportunité croît assez vite car des agriculteurs de moins en moins performants participent à l'offre. Dès 700000 tonnes de colza ester, quand la substitution avec le colza alimentaire se manifeste, le coût d'opportunité se stabilise mais à un niveau légèrement inférieur à celui du prix du colza alimentaire en raison de la prime de 45 euros.

Ce coût d'opportunité indique le prix minimum du colza énergétique qui va inciter les producteurs à offrir la quantité demandée. $\mathrm{Ce}$ renchérissement se répercute sur le coût d'opportunité de l'ester (figure 3) sans pour autant se répercuter obligatoirement sur le coût réel ${ }^{3}$. Ce dernier peut rester stable si le prix réel du colza énergétique entrant dans le calcul du coût réel est suffisamment élevé pour absorber la hausse du coût d'opportunité du colza ester. Dans ce cas, on assistera seulement à une baisse du revenu agricole moyen additionnel par quintal de graines de colza. C'est la situation actuelle où le prix du colza énergétique est proche du prix du colza alimentaire en raison des prix élevés du pétrole.

Effets des variantes $B, C, D$ sur l'offre de colza alimentaire (figure 4)

Le scénario $A$ en gras sur le graphique est le scénario central ; dans le scénario B, la prime énergétique du colza énergétique produit dans l'assolement alimentaire est supprimée ; dans le scénario C, le prix du colza baisse et passe de 19,8 euros/quintal en 2004 à 18 euros en 2010 ; enfin, dans le scénario $D$, la jachère est supprimée. Dans tous les cas, les évolutions de la production de colza non alimentaire et aussi de blé et de betterave éthanol restent identiques ainsi que les évolutions des prix des céréales (baisse des prix de 9,2 à 8,6 euros par quintal).

\footnotetext{
$\overline{{ }^{3} \text { Lorsque le }}$ coût réel de l'ester est supérieur à son coût d'opportunité, le soutien public peut en principe être réduit de manière à rapprocher le coût réel et le coût d'opportunité et à diminuer ainsi une partie des rentes existantes dans la filière.
} 


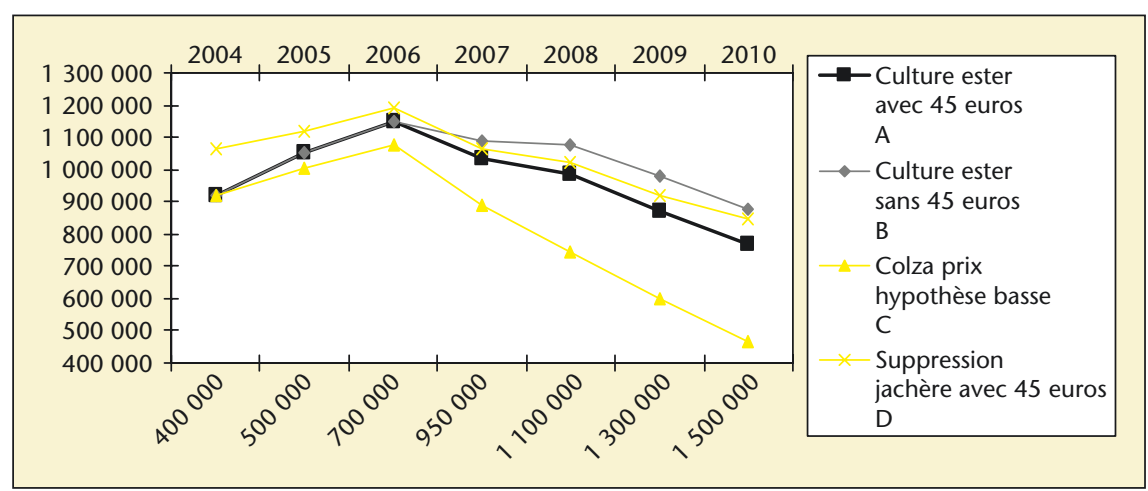

Figure 4. Evolution des surfaces en colza alimentaire selon les scénarios.

Les évolutions des courbes de surface en colza montrent deux phases : jusqu'en 2006, la surface de colza alimentaire s'accroît quels que soient les scénarios. Rappelons les facteurs qui interviennent : l'effet des réformes de la PAC et de I'OCM sucre, l'amélioration de la compétitivité du colza par rapport aux céréales et l'absence de concurrence du colza non alimentaire.

Au-delà de 2006, les surfaces en colza alimentaire diminuent. Le scénario $D$, évolution défavorable des prix du colza, est celui qui affecte le plus I'offre de colza alimentaire; les surfaces sont quasiment divisées par deux entre 2004 et 2010. À l'opposé, le scénario B, suppression de la prime de 45 euros par hectare, est celui qui freine le plus la baisse des surfaces en colza alimentaire. En 2010, on retrouve les surfaces de 2004.

Le scénario $D$, suppression de la jachère, translate en quelque sorte la courbe du scénario central vers le haut. L'effet sur les surfaces en colza $n^{\prime}$ est pas considérable car $1 / 3$ des exploitations de l'échantillon n'ont pas de colza et ne sont pas autorisées à en produire, le colza est soumis à une contrainte agronomique l'empêchant de dépasser $30 \%$ des surfaces, beaucoup d'exploitations atteignent cette limite et ne peuvent donc pas profiter de la remise en culture de la jachère pour accroître leur surface en colza, enfin 350000 hectares de jachère sont difficilement réutilisables. Le scénario $D$ et le scénario B (suppression des 45 euros de prime) donnent à peu près les mêmes surfaces en colza en 2010.

\section{Conclusion}

Les simulations du modèle OSCAR développé par I'INRA montrent que I'objectif 2007
(950 000 tonnes de colza ester) commence à induire une compétition entre le colza non alimentaire et alimentaire. Cette compétition précoce est due à la présence d'une proportion significative de jachère non cultivable ( $25 \%$ ), à la présence de blé éthanol et de betterave éthanol qui vont en majeure partie sur la jachère et de la prime de 45 euros par hectare qui favorise le transfert du colza énergétique vers I'assolement alimentaire. Au-delà de 2007, les surfaces en colza alimentaire peuvent baisser de manière importante. En 2010, le scénario central donne une surface en colza alimentaire inférieure à la surface initiale 2004, alors que les rapports de prix « colza céréales » sont plutôt favorables au colza : les prix des céréales baissent en euros courants alors que les prix du colza s'accroissent légèrement (voir tableau 1). Si l'accroissement de la production non alimentaire s'accompagne d'une diminution des prix du colza alimentaire, la chute des surfaces de colza alimentaire est alors considérable (scénario D). Ce scénario est sans doute peu probable dans la mesure où le prix du colza alimentaire risque plutôt $d^{\prime}$ augmenter en raison de la diminution de l'offre. Le modèle OSCAR, ne prenant pas en compte pour l'instant les fonctions de demande en colza alimentaire, ne peut donc simuler les évolutions de prix.

Autre inconvénient de cette compétition, le renchérissement du colza ester et de l'ester de colza sous la forme d'un accroissement des coûts d'opportunité. En pratique, ce renchérissement peut passer inaperçu si le prix effectif du colza ester est suffisamment élevé mais il affecte la compétitivité macroéconomique (ou publique) de l'ester qui est toujours très discutée.
Enfin, on peut s'interroger sur la répartition des agréments décidés entre éthanol et ester, compte tenu notamment de la jachère qui devient rapidement le facteur limitant du colza (ce qui est moins le cas pour le blé, encore moins pour la betterave). Cet équilibre esteréthanol reflète-t-il une utilisation optimale des fonds publics, du point de vue agricole, du point de vue de la réduction des émissions de gaz à effets de serre, du point de vue des coûts pour satisfaire les objectifs européens de production de biocarburants? Ces questions feront l'objet des prochaines interrogations du modèle OSCAR.

\section{RÉFÉRENCES}

COSTA S. Analyse coûts-avantages d'une production d'ester méthylique de colza. INRA Etudes Economiques $n^{\circ} 23,50 p, 1995$.

Directive 2003/30/CE du Parlement Européen et du Conseil du 8 mai 2003 visant à promouvoir l'utilisation de biocarburants ou autres carburants renouvelables dans les transports. Journal Officiel de I'Union Européenne; L123-42 à L123-46, mai 2003.

Directive 2003/96/CE du Conseil du 27 octobre 2003 restructurant le cadre communautaire de taxation des produits énergétiques et de l'électricité. Journal Officiel de I'Union Européenne. L283/51 à L283/70, octobre 2003.

GUESNERIE R, et al. Kyoto et l'économie de l'effet de serre, Rapport du Conseil d'Analyse Economique, La Documentation Française, 266 p., 2003.

PASTY IC, et al. Les débouchés non alimentaires des produits agricoles : un enjeu pour la France et I'Union Européenne, Rapport du Conseil Economique et Social, 184 p., 2004.

PREVOT H. Est-il possible de diminuer profondément les émissions de gaz à effet de serre liées à l'énergie? Un scénario de division par trois en trente ans. Conditions de réalisation, conséquences, Conseil Général des Mines, 30 p, 2004.

ROZAKIS S, SOURIE JC. Micro-economic modelling of biofuel in France to determine tax exemption policy under uncertainty, Energy Policy, 12p, 2005.

ROZAKIS S, SOURIE IC, VANDERPOOTEN D. Integrated micro-economic modelling and multi-criteria methodology to support public decision-making: the case of liquid bio-fuels in France, Biomass and Bioenergy 20: 385-398, 2001.

SOURIE JC, ROZAKIS S. Bio-fuel production system in France: an Economic Analysis, Biomass and Bioenergy 20: 483-489, 2001

SOURIE JC. Agricultural raw materials cost and supply for bio-fuel production : methods and concepts, options méditerranéennes, série $A n^{\circ}$ 48, 2002. 